\title{
Distribution of methyl substituents over crystalline and amorphous domains in methylated starches
}

\author{
Yuri E.M. van der Burgt ${ }^{a}$, Jack Bergsma ${ }^{b}$, Ido P. Bleeker ${ }^{b}$, Paul J.H.C. Mijland ${ }^{b}$,
} Anca van der Kerk-van Hoof ${ }^{c}$, Johannis P. Kamerling ${ }^{a, *}$, Johannes F.G. Vliegenthart ${ }^{a}$

${ }^{a}$ Bijvoet Center, Department of Bio-Organic Chemistry, Utrecht University, PO Box 80.075, NL-3508 TB Utrecht, The Netherlands

${ }^{\mathrm{b}}$ AVEBE Research \& Development, AVEBE-weg 1, NL-9607 PT Foxhol, The Netherlands

c Bijvoet Center, Department of Mass Spectrometry, Utrecht University, Sorbonnelaan 16, NL-3584 CA Utrecht, The Netherlands

Received 18 March 1999; accepted 28 May 1999

\begin{abstract}
Granular potato starch and amylopectin potato starch were methylated in aqueous suspension with dimethyl sulfate to molar substitution (MS) up to 0.29. The percentage of amorphous starch compared with crystalline domains increased with increasing MS. Prolonged treatment of these methylated starches with hydrochloric acid below the swelling temperature resulted in the release of D-glucose and small D-glucose-oligomers from the amorphous domains. The granular structure was maintained during the acidic treatment, indicating that the crystalline lamellae were less affected by acid. The amorphous domains contained on average about twice as many substituents per glucose unit as the remaining crystalline network. The distributions of methyl substituents in trimers and tetramers, released from amorphous domains and prepared from crystalline fractions, were determined by FABMS and compared to the outcome of a statistically random distribution. Quantification of the degree of heterogeneity of the thus-obtained trimers and tetramers showed a much larger deviation from random substitution in the crystalline fractions compared with the amorphous ones. These results are in agreement with our previous study that describes substitution patterns in branched and linear regions of methylated starch granules. (C) 1999 Elsevier Science Ltd. All rights reserved.
\end{abstract}

Keywords: Methylated starches; Amylopectin potato starch; Lintnerisation; Substituent distribution

\section{Introduction}

Starch is an important renewable raw material that is used in food, pharmaceuticals, and technical and paper products. In higher plants starch is deposited as a mixture of amylose and amylopectin in cold-water insoluble, partially crystalline granules [1]. The size $(1-100$

* Corresponding author. Tel.: + 31-30-253-3479; fax: + 31-30-254-0980.

E-mail address: kame@boc.chem.uu.nl (J.P. Kamerling) $\mu \mathrm{m})$ and shape of these granules vary for each plant species. Granules consist of alternating shells of amorphous and crystalline material [2], the latter made up of double-helically arranged short side chains of amylopectin, oriented perpendicular to the shell (Fig. 1) [3].

Recently, evidence for the presence of alternating shells in individual starch granules was obtained by scanning different areas with 2 $\mu \mathrm{m}$ wide synchrotron X-ray beams [4-7]. Generally, cereal starches exhibit A-type diffraction patterns, whereas tuber starches usu- 
ally yield B-type patterns [8], resulting from differences in packing of amylopectin side chains [9]. The degree of crystallinity is dependent on factors such as plant origin, amylose/ amylopectin ratio and moisture content $[10,11]$. Amylose is considered to be an essentially linear $(1 \rightarrow 4)$ - $\alpha$-D-glucan, located mainly in the amorphous domains of the starch granules [12]. Amylopectin is a $(1 \rightarrow 6)$ branched $(1 \rightarrow 4)$ - $\alpha$-D-glucan with a molecular mass approximately $10^{2}-10^{4}$ times higher than that of amylose. Enzymatic and X-ray studies have shown that branching points, linking short-, long-, and average-length $(1 \rightarrow 4)$-chains, are not distributed at random $[13,14]$, but are located in the disordered amorphous domains between the crystallites $[15,16]$. The supramolecular model of amylopectin [17], in which parts of the double-helical side chains participate in a continuous, crystalline network that functions as a skeleton for all other starch chains, still needs more experimental evidence.

Modifications of starches are carried out in order to provide starch products with properties needed for specific uses. In most chemical derivatisations of starch hydroxyl groups are partially substituted [18]. The number, location and distribution of the substituents not expected to be random in view of the different levels of organisation within the starch granule [19] — determine the properties of these starch derivatives. In order to acquire knowledge about the relationship between structure and function, it is important to determine these parameters after derivatisation. Recently, we have reported that in

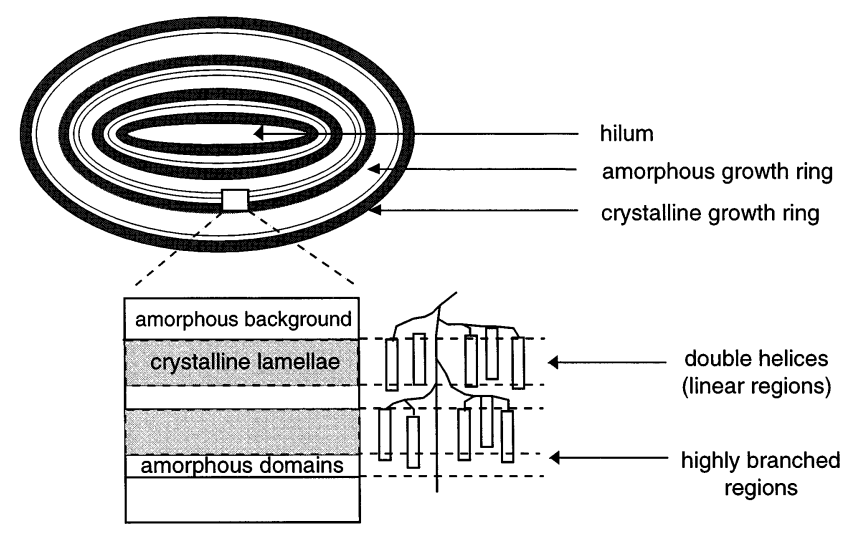

Fig. 1. Schematic model of a starch granule. methylated starches branched regions of amylopectin contain two to three times more substituents than linear regions of amylopectin [20]. This observation can be explained on the basis of the crystalline framework as shown in Fig. 1, wherein linear side chains of amylopectin appear as double helices in crystalline domains, therefore probably being less accessible for chemical substitution.

The aim of this study is to obtain structural information on the substitution differences in crystalline and amorphous domains of methylated potato starch and methylated amylopectin potato starch. Crystalline and amorphous domains both affect the gelatinisation behaviour of starch: the former shows melting behaviour in heating processes, whereas the latter undergoes glass-rubber transitions. After separation of these domains, the level of substitution and the distribution of substituents along the polymer have been determined. These results, in combination with our earlier study of branched and linear regions [20], provide more detailed information with respect to the distribution profile of methyl substituents in methylated starch granules.

\section{Results and discussion}

Preparation of methylated starches.Methylated potato starch and methylated amylopectin potato starch were prepared by methylation of starch granules in an aqueous suspension using dimethyl sulfate. The molar substitution (MS) values of the studied starch derivatives P10, P30, A10 and A30 with varying degrees of methylation are listed in Table 1. The determination of these MS values and the monomer compositions of P10, P30, A10 and A30 have been reported previously [20].

Isolation of amorphous and crystalline fractions. - Methylated starch granules (P10, P30, A10, A30) were treated below the swelling temperature $\left(30^{\circ} \mathrm{C}\right)$ with $2.2 \mathrm{M} \mathrm{HCl}$, resulting in the release of small (partially methylated) D-glucose-oligomers (degree of polymerisation (DP), mainly 2-4); HPLC analysis (data not shown)) and D-glucose from the amorphous domains [21-23]. The remaining insoluble 
Table 1

Molar substitution (MS) values of amorphous and crystalline fractions from methylated potato starch and amylopectin potato starch

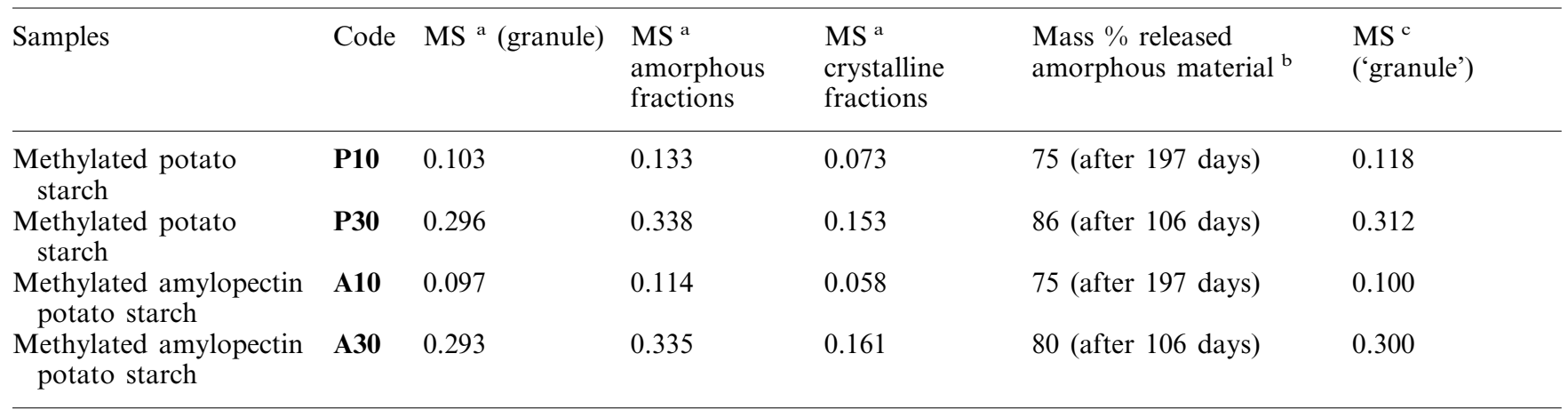

${ }^{\text {a }} \mathrm{MS}$ is defined as mol of substituents/mol of Glc monomer. The MS varies from 0 (native starch) to 3 (permethylated linear starch) and is measured via monosaccharide analysis (GLC) in triplo $(\sigma<0.01)$ [20].

b Steady-state level of dissolved carbohydrate, as determined by the anthrone method (Fig. 2).

${ }^{\mathrm{c}} \mathrm{MS}$ values of virtual granules, reconstructed from amorphous and crystalline fractions, calculated according to $\mathrm{MS}\left(\right.$ 'granule') $=(\text { percentage MS })_{\text {amorphous fractions }}+(\text { percentage MS })_{\text {crystalline fractions }}$.

residues are usually referred to as lintnerised starches that consist of acid-resistant crystalline parts of amylopectin. Recently, the preferential destruction of amorphous domains (i.e., etching away non-crystalline glucans) during hydrolysis was confirmed by $\mathrm{X}$-ray scattering studies and differential scanning calorimetry [24]. This feature can be rationalised in view of the narrower and more constrained crystalline lamellae that are less susceptible to acid hydrolysis. All samples were incubated for 197 days, but for preparative purposes lintnerisation of P30 and A30 was stopped after 106 days (Fig. 2). The mixtures of glucose and glucose-oligomers in solution were separated from the granules in suspension by centrifugation, then neutralised and desalted. The solubilised carbohydrate mixtures will be referred to as the 'amorphous fractions' and the remaining lintnerised granules as the 'crystalline fractions'. Inspection of the lintner residues of P10, P30, A10 and A30 under polarised light still showed a Maltese cross after 108 days of acidic treatment, suggesting that the crystallinity of the granules was maintained despite the fact that $60-85 \%$ of all glucans was dissolved from the granules (Fig. 3) [2].

Acidic treatment of potato starch (P0) and amylopectin potato starch (A0) for 197 days resulted in the release of carbohydrate that levelled off at values between 50 and $60 \%$
(Fig. 2). The level of dissolved carbohydrate was higher for methylated starches. Apparently, methylation of starch decreases the degree of crystallinity. These results indicate that the number of amylopectin side chains, present in ordered crystalline domains (Fig. $1)$, decreases with increasing MS. The coldwater swellability and solubility that is observed at MS values above 0.5 may be attributed to a total loss of crystallinity. It is

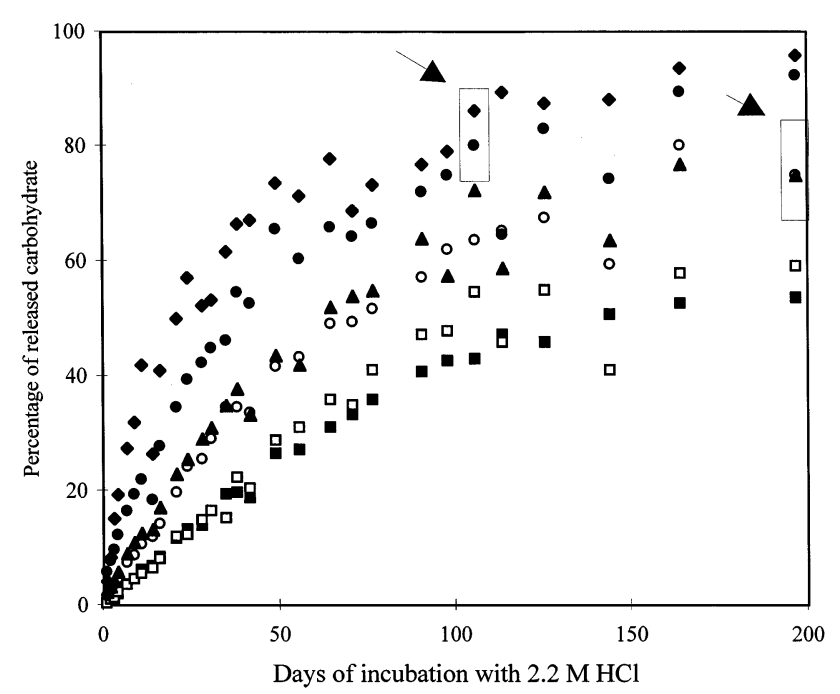

Fig. 2. Amount of released carbohydrate material from amor-

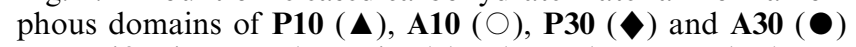
at specific times as determined by the anthrone method [25]. Native potato starch and amylopectin potato starch (P0 ( $\square$ ) and $\mathbf{A 0}(\mathbf{\square}), \mathrm{MS}=0)$ were used as references. The arrows indicate the time at which preparative lintnerisation was stopped to isolate amorphous and crystalline fractions. 


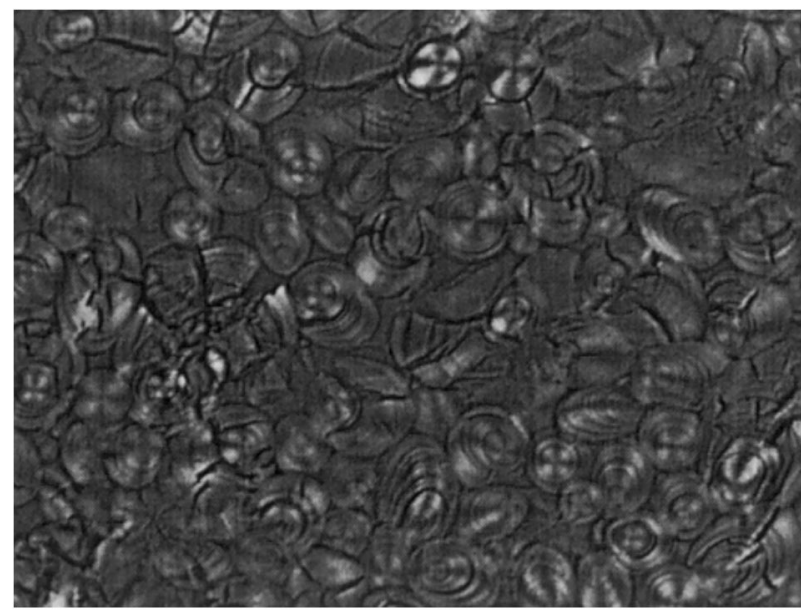

Fig. 3. Partially methylated starch granules (P30), after lintnerisation (108 days of incubation, 2.2 M HCl).

clear from Fig. 2 that the rate of release increases with increasing MS, suggesting relatively easier hydrolysis of partially methylated carbohydrate.

Molar substitutions of amorphous and crystalline fractions from methylated starches.The MS values of the amorphous and the crystalline fractions of P10, P30, A10 and A30 were measured by monosaccharide analysis $[20,26]$. The monomer compositions and derived MS values are given in Tables 2 and 1, respectively.

It is clear from Table 1 that in each sample amorphous fractions roughly contain twice as many methyl substituents as the crystalline ones. In this respect no differences are observed between methylated potato starch (P10 and P30) and methylated amylopectin potato starch (A10 and A30). This preference for substitution of amorphous domains during chemical derivatisation is in agreement with our earlier observation of higher substitution at branched regions [20], which are, according to the current model of the starch granule (Fig. 1), located in amorphous domains. The calculated MS value of the 'granule' (virtual granule reconstructed from amorphous and crystalline fractions) deviates only slightly from the measured MS of the intact granule, indicating that no material was lost during lintnerisation. From Table 2 it is clear that in all methylated starches the HO-2 group has by far the highest positional degree of substitution and that ratios between substituted monomers for each sample are constant. Therefore, no topochemical effects can be demonstrated on the level of monosaccharides, which is in agreement with earlier reports [12,20].

Distribution of substituents in methylated amorphous domains and methylated crystalline domains. - The distribution of substituents in oligomer fragments represents the substitution pattern in the corresponding polymer backbone [27,28]. In order to prepare suitable derivatives for FAB mass spectrometric analysis, the amorphous fractions derived from P10, P30, A10 and A30, containing glucose and glucose-oligomers (DP mainly 2-4), were permethylated with $\mathrm{CD}_{3} \mathrm{I}$ [29]. It can be anticipated that the thus-obtained per(deuterio)methylated oligomers with the same DP, only varying in number of $\mathrm{CH}_{3}$ - and $\mathrm{CD}_{3}$-substituents, show the same behaviour in terms of ionisation because of their chemical equivalency (Fig. 4). Therefore, quantification of all FAB intensities in a pseudo-molecular-ion region $\left(\left[\mathrm{M}+\mathrm{Na}^{+}\right]\right)$of oligomers with the same $\mathrm{DP}$ is valid. In this respect trimers and te-

Table 2

Monomer compositions of amorphous (a) and crystalline (c) fractions from methylated potato starch (P10, P30) and methylated amylopectin potato starch (A10, A30)

\begin{tabular}{lccccccc}
\hline Samples & Glc & Glc2Me & Glc3Me & Glc6Me & Glc2,3Me & Glc2,6Me $_{2}$ & Glc3,6Me $_{2}$ \\
\hline P10 (a) & 87.5 & 8.3 & 1.9 & 1.5 & 0.4 & 0.3 & 0.1 \\
P10 (c) & 93.1 & 4.4 & 1.3 & 0.8 & 0.1 & 0.2 & 0.1 \\
P30 (a) & 70.2 & 18.9 & 4.3 & 2.6 & 2.0 & 0.4 & 0.4 \\
P30 (c) & 85.7 & 9.6 & 2.3 & 1.4 & 0.4 & 0.3 & 0.1 \\
A10 (a) & 89.4 & 6.2 & 2.2 & 1.4 & 0.1 & 0.1 \\
A10 (c) & 94.4 & 3.6 & 1.0 & 0.8 & 2.1 & 0.5 & 0.4 \\
A30 (a) & 70.5 & 18.7 & 4.2 & 2.6 & 0.6 & 0.1 \\
A30 (c) & 85.1 & 10.0 & 2.3 & 1.4 & & \\
\hline
\end{tabular}

\footnotetext{
${ }^{a}$ All monomers are characterised as trimethylsilylated methyl glucopyranosides $(\alpha+\beta)$ and are given in molar percentages.
} 


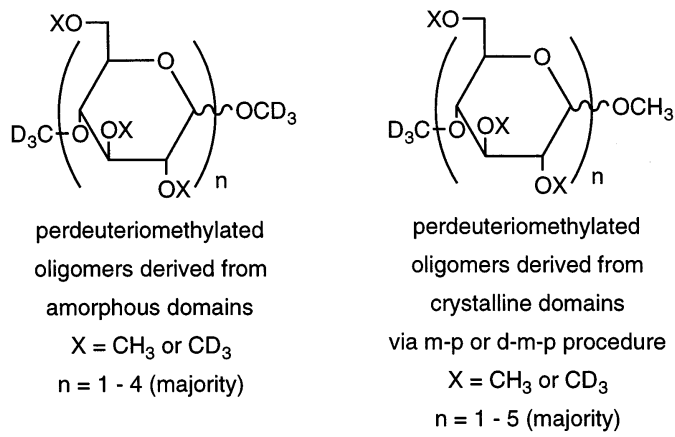

Fig. 4. Synthesised perdeuteriomethylated oligomers.

tramers are of specific interest for FAB mass spectrometric analysis, because resolution decreases using larger oligomers.

Substituent distributions in crystalline domains of P10, P30, A10 and A30 were analysed after preparing suitable derivatives in two different ways:

1. Partial methanolysis of crystalline fractions followed by perdeuteriomethylation (m-p procedure).

2. Deuteriomethylation of crystalline fractions followed by partial methanolysis, followed by perdeuteriomethylation (d-m-p procedure).

In Table 3 the MS values of all trimers and tetramers obtained from amorphous and crystalline fractions are listed, as determined by FABMS.

It is clear from Table 3 that the MS values of the trimers and tetramers in the amorphous fractions of P10, P30, A10 and A30, as deter- mined from FAB mass spectrometric intensities, differ by less than $10 \%$ from the overall MS values of the amorphous fractions (measured via monosaccharide analysis). Therefore, these trimers and tetramers represent substituent distributions in amorphous domains. The situation is more complex for oligomers derived from crystalline fractions, obtained via the m-p or the d-m-p procedure. Here, differences between the MS values of the oligomers and the overall substitution levels of crystalline domains result from heterogeneous $\mathrm{CH}_{3}$-group distributions. MS values of trimers and tetramers, obtained via the $\mathrm{m}-\mathrm{p}$ procedure, all show relatively large differences. Both negative and positive deviations are found, which can be interpreted as a random release of oligomers from the crystalline domains. The $d-m-p$ procedure results in oligomers that all have lower MS values than the overall substitution levels of crystalline domains. Apparently, areas with high $\mathrm{CH}_{3}$ substitution are perdeuteriomethylated during the first methylation, whereas areas with low $\mathrm{CH}_{3}$-substitution (with difficult accessibility) remain under-deuteriomethylated. Partial methanolysis of the per(deuterio)methylated areas results in a quick release of glucose with a relative excess of $\mathrm{CH}_{3}$-substitution (here, methanolysis is more efficient), whereas the under-deuteriomethylated areas with relatively low $\mathrm{CH}_{3}$-substitution slowly release oligomers. Therefore, trimers and tetramers thus ob-

Table 3

MS values of trimers and tetramers derived from amorphous and crystalline domains of P10, P30, A10 and A30

\begin{tabular}{|c|c|c|c|c|}
\hline Samples & P10 & P30 & A10 & A30 \\
\hline Overall MS ${ }^{\text {a }}$ amorphous fractions & 0.133 & 0.338 & 0.114 & 0.335 \\
\hline $\mathrm{MS}^{\mathrm{b}}$ amorphous fractions (trimers) & $0.137(+3.0)^{\mathrm{c}}$ & $0.324(-4.1)$ & $0.125(+9.6)$ & $0.327(-2.4)$ \\
\hline Overall $\mathrm{MS}^{\mathrm{b}}$ crystalline fractions & 0.073 & 0.153 & 0.058 & 0.161 \\
\hline 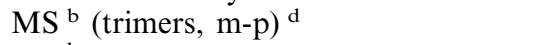 & $0.067(-8.2)$ & $0.228(+49)$ & $0.062(+6.9)$ & $0.221(+37)$ \\
\hline 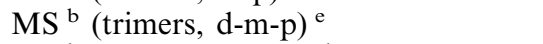 & $0.052(-29)$ & $0.144(-5.9)$ & $0.046(-21)$ & $0.143(-11)$ \\
\hline
\end{tabular}

\footnotetext{
${ }^{a}$ Measured via monosaccharide analysis (GLC).

${ }^{\mathrm{b}}$ Determined from FABMS intensities, e.g., for trimers: [mole fraction $\operatorname{DS}(0) \cdot 0+$ mole fraction $\operatorname{DS}(1) \cdot 1+\ldots+$ mole fraction $\operatorname{DS}(9) \cdot 9] / 3$.

c The difference between the overall MS value of the amorphous fraction and the MS value of the trimers or tetramers is indicated in percentages between parentheses. The same is done for crystalline fractions and derived oligomers.

${ }^{\mathrm{d}}$ Oligomers obtained after partial methanolysis of crystalline fractions followed by perdeuteriomethylation.

e Oligomers obtained after deuteriomethylation of crystalline fractions, followed by partial methanolysis and subsequent perdeuteriomethylation.
} 


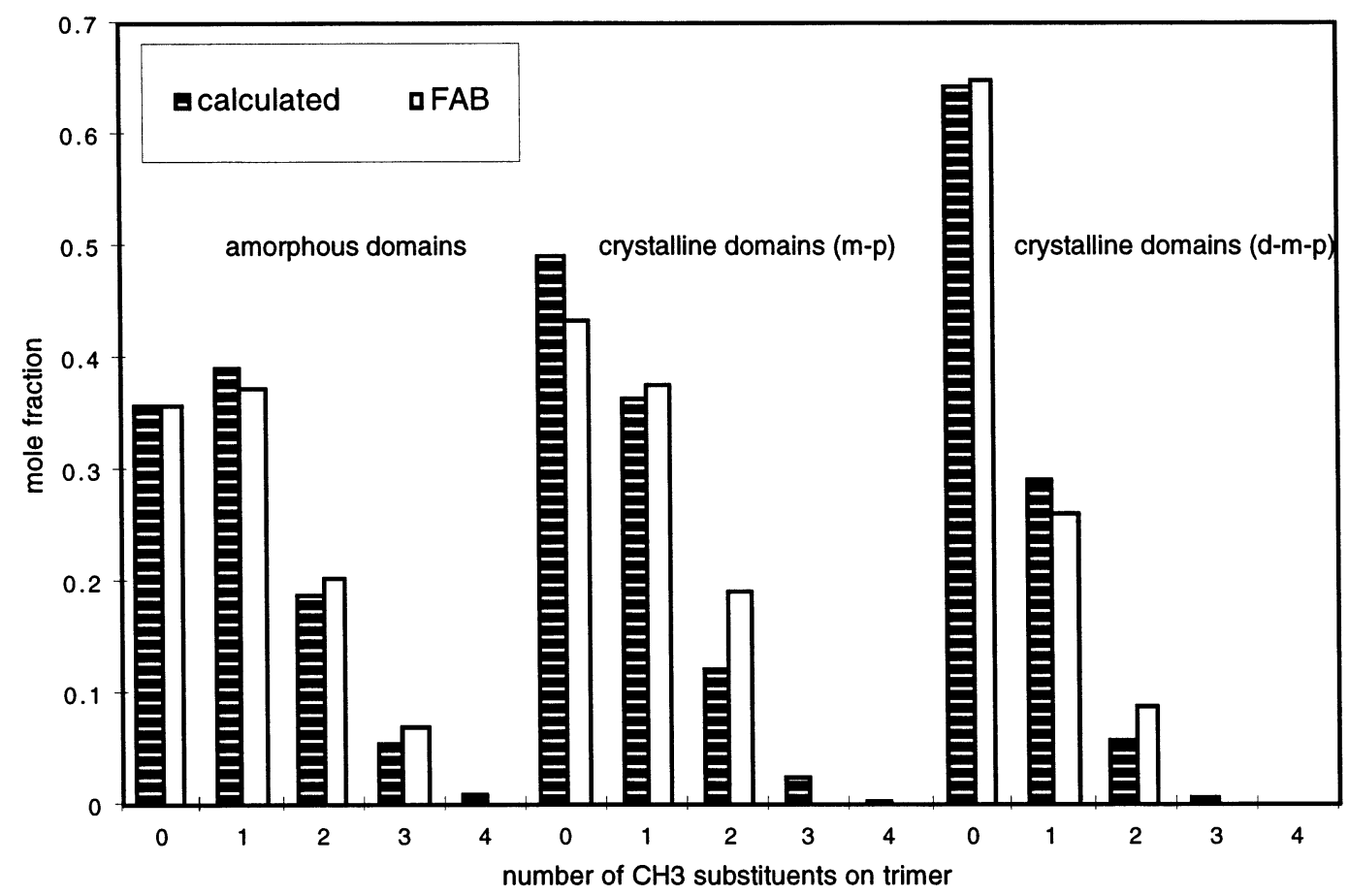

Fig. 5. Calculated and observed substituent distribution in trimers from amorphous domains (left) and crystalline domains (middle and right) of P30. Note that the amorphous domains contain more substituents than the crystalline ones (m-p $=$ trimers obtained after partial methanolysis of crystalline fractions followed by perdeuteriomethylation; $\mathrm{d}-\mathrm{m}-\mathrm{p}=$ trimers obtained after deuteriomethylation of crystalline fractions, followed by partial methanolysis and subsequent perdeuteriomethylation).

tained represent relatively low substituted areas in crystalline domains.

The FAB mass spectrometric data of oligomers derived from amorphous and crystalline domains ( $m-p$ and $d-m-p$ procedure) were compared with the expected distributions according to binomial statistics. In the calculation $\mathrm{CH}_{3}$-substituents are presumed to be distributed randomly over equal and independent glucose monomers. A typical example for trimers derived from P30 is shown Fig. 5.

For the amorphous domains the observed contribution of di- and trisubstituted oligomers is slightly higher than expected on the basis of a random distribution, whereas the amount of monosubstituted oligomers is lower. These differences are more pronounced in the crystalline domains. This is direct evidence for a more heterogeneous substitution of the crystalline domains during methylation of granular starch. In order to visualise these differences in a more quantitative way, the degree of heterogeneity is expressed in the parameter $H_{n}$ where $n$ corresponds with the DP of the per(deuterio)methylated oligomers [27]. All heterogeneity values in Table 4 are normalised by dividing by the MS value of the corresponding trimers or tetramers. Only in this way can heterogeneities of samples with different degrees of substitution be compared. If there are no differences between observed and calculated mole fractions (Fig. 5), the $\mathrm{CH}_{3}$-substituent distribution is homogeneous $\left(H_{n}=0\right)$. With increasing differences between observed and calculated mole fractions the values of $H_{n}$ increase. It is evident from Table 4 that $\mathrm{CH}_{3}$-substituents in amorphous domains are closer to a random-like distribution (i.e., according to binomial statistics) than the crystalline domains, where higher degrees of heterogeneity are observed. Both trimer and tetramer fractions show these results.

\section{Conclusions}

In this study it is shown that methylation of starch in a granular suspension takes place preferably at the amorphous domains, whereas crystalline parts of amylopectin (linear side chains) contain less methyl substituents. However, substituents in crystalline 
Table 4

Heterogeneity of substituent distribution in methylated potato starch and amylopectin potato starch

\begin{tabular}{|c|c|c|c|c|}
\hline & P10 & P30 & A10 & A30 \\
\hline$H_{3} / \mathrm{MS}\left(\right.$ trimers) $(\mathrm{a})^{\mathrm{a}, \mathrm{b}}$ & 0.210 & 0.214 & 0.186 & 0.234 \\
\hline$H_{3} / \mathrm{MS}\left(\right.$ trimers, m-p) $(\mathrm{c})^{\mathrm{c}}$ & 1.209 & 0.501 & 1.290 & 0.482 \\
\hline $\begin{array}{l}H_{3} / \mathrm{MS}(\text { trimers, d-m-p) } \\
\text { (c) }{ }^{\mathrm{d}}\end{array}$ & 1.083 & 0.319 & 1.203 & 0.315 \\
\hline$H_{4} / \mathrm{MS}($ tetramers) (a) & 0.446 & 0.285 & 0.390 & 0.296 \\
\hline$H_{4} / \mathrm{MS}$ (c) & 3.787 & 1.047 & 3.849 & 1.021 \\
\hline $\begin{array}{l}H_{4} / \mathrm{MS} \text { (tetramers, d-m-p) } \\
\text { (c) }\end{array}$ & 1.982 & 0.632 & 2.179 & 0.632 \\
\hline
\end{tabular}

a (a) amorphous domains; (c) crystalline domains.

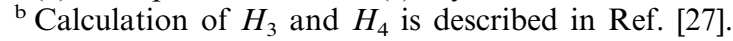

c Trimers, $\mathrm{m}-\mathrm{p}=$ trimers obtained after partial methanolysis of crystalline fractions followed by perdeuteriomethylation.

d Trimers, $d-m-p=$ trimers obtained after deuteriomethylation of crystalline fractions, followed by partial methanolysis and subsequent perdeuteriomethylation.

domains are distributed more heterogeneously than those in amorphous domains. In a previous paper we reported that a similar methylation of starch granules resulted in the preferential substitution of branched regions if compared with linear chains of amylopectin [20]. It was found that branched regions were substituted nearly at random. All these results are in excellent agreement with the current model of a starch granule, in which branched regions are located in the amorphous (sub)domains of starch $[4,15,16]$.

\section{Materials and methods}

Preparation of methylated starches. - Potato starch granules and amylopectin potato starch granules were suspended in water and methylated using dimethyl sulfate at constant temperature $\left(20^{\circ} \mathrm{C}\right)$ and $\mathrm{pH}(11)$ for $4-6 \mathrm{~h}$ (Table 1) [30]. Samples with different MS values were obtained by varying the amount of dimethyl sulfate added to the granular suspension (Table 1).

Partial acid hydrolysis of methylated starch granules.-Methylated starches were suspended in $2.2 \mathrm{M} \mathrm{HCl}$ and partially hydrolysed at $30{ }^{\circ} \mathrm{C}$. The mixture of glucose and oligoglucans released from the acid-treated granules was isolated after homogenisation and centrifugation. The residual granules were sepa- rated from $\mathrm{HCl}$ by repeated centrifugation and washing the samples with demineralised water (at least five times). Carbohydrate-containing fractions were pooled and dried under vacuo. The quantification of dissolved carbohydrate in amorphous fractions obtained was carried out using an anthrone assay with glucose as a standard [25]. Before the determination of MS values and monomer analysis, released carbohydrate fractions were neutralised with $\mathrm{NaOH}$, then desalted on an anion-exchange column $\left(\mathrm{OH}^{-}\right.$form, IMAC A24).

Analytical procedures.-Monosaccharide analysis was carried out by subjecting partially methylated oligo- or polyglucan samples to methanolysis (methanolic $1 \mathrm{M} \mathrm{HCl}, 18 \mathrm{~h}$, $85^{\circ} \mathrm{C}$ ). The resulting mixtures of methyl glucoside derivatives were trimethylsilylated (1:1:5 hexamethyldisilazane-trimethylchlorosilane-pyridine), identified by GLC-MS [26], and quantified by GLC using empirical molar response factors [20]. GLC analyses were performed on a WCOT CP-SIL 5CB fused-silica capillary column $(25 \mathrm{~m} \times 0.32 \mathrm{~mm})$ using a temperature program of $110-230^{\circ} \mathrm{C}$ at $4{ }^{\circ} \mathrm{C} /$ min. GLC-MS of ( $O$-methylated) glucose derivatives, measured as trimethylsilylated methyl glucosides, was carried out on an MD800/8060 system (Fisons Instruments; electron energy, $70 \mathrm{eV}$ ), equipped with a DB-1 fused-silica capillary column $(30 \mathrm{~m} \times 0.32$ $\mathrm{mm}, \quad J \& W$ Scientific), using a temperature program of $110-230^{\circ} \mathrm{C}$ at $4{ }^{\circ} \mathrm{C} / \mathrm{min}$. Partially methylated oligoglucans from amorphous domains were permethylated with $\mathrm{CD}_{3} \mathrm{I}$ as described previously $\left(\mathrm{Me}_{2} \mathrm{SO} / \mathrm{NaOH}\right)$ [29], and analysed by FABMS. The same permethylation procedure was followed for partially methylated oligoglucans from crystalline domains, obtained in two different ways after partial methanolysis $\left(3 \mathrm{~h}, 60^{\circ} \mathrm{C}\right.$, methanolic $0.5 \mathrm{M} \mathrm{HCl}$; see Section 2). FABMS was performed on a Jeol JMS SX/SX 102A four-sector mass spectrometer, operated at $10 \mathrm{kV}$ accelerating voltage, equipped with a Jeol MSFAB $10 \mathrm{D}$ FAB gun operated at a $10 \mathrm{~mA}$ emission current, producing a beam of $6 \mathrm{keV}$ xenon atoms. The per(deuterio)methylated oligosaccharide samples were measured over a mass range of $m / z^{10-1200 ~ i n ~ a ~ m a t r i x ~ o f ~}$ 
$m$-nitrobenzyl alcohol saturated with NaI, using the standard resolution of 1500 . The intensities of the sodium-cationised oligosaccharide ions were determined from their FAB spectra and compared with the statistically expected ones. The statistically expected ratios between non-, di-, tri-, tetra- and penta-substituted oligomers with specific DP were calculated according to a random distribution of $\mathrm{CH}_{3}$ substituents at given overall $\mathrm{MS}$ value. The heterogeneity parameter $H_{n}$ is then calculated from the sum of all absolute differences between determined and expected intensities of each oligomer.

\section{Acknowledgements}

This study was supported by the PBTS Research Program with financial aid from the Ministry of Economic Affairs and the Integral Structure Plan for the Northern Netherlands from the Dutch Development Company.

\section{References}

[1] S.G. Ring, M.J. Miles, V.J. Morris, R. Turner, P. Colonna, Int. J. Biol. Macromol., 9 (1987) 158-160.

[2] D. French, in R.L. Whistler, J.N. BeMiller, E.F. Paschall (Eds.), Starch: Chemistry and Technology, second ed., Academic Press, London, 1984, pp. 183-247.

[3] A. Imberty, S. Pérez, Biopolymers, 27 (1988) 1205-1221.

[4] P.J. Jenkins, R.E. Cameron, A.M. Donald, W. Bras, G.E. Derbyshire, G.R. Mant, A.J. Ryan, J. Polym. Sci. Polym. Phys. Ed., 32 (1994) 1579-1583.

[5] T.A. Waigh, I. Hopkinson, A.M. Donald, M.F. Butler, F. Heidelbach, C. Riekel, Macromolecules, 30 (1997)
$3813-3820$.

[6] A. Buléon, B. Pontoire, C. Riekel, H. Chanzy, W. Helbert, R. Vuong, Macromolecules, 30 (1997) 3952-3954.

[7] P. Calvert, Nature, 389 (1997) 338-339.

[8] H.F. Zobel, Starch/Stärke, 40 (1988) 1-7.

[9] A. Imberty, H. Chanzy, S. Pérez, A. Buléon, V. Tran, Macromolecules, 20 (1987) 2634-2636.

[10] S. Nara, T. Komiya, Starch/Stärke, 35 (1983) 407-410.

[11] H.F. Zobel, Starch/Stärke, 40 (1988) 44-50.

[12] P.A.M. Steeneken, A.J.J. Woortman, Carbohydr. Res., 258 (1994) 207-221.

[13] S. Hizukuri, Y. Takeda, N. Maruta, B.O. Juliano, Carbohydr. Res., 189 (1989) 227-235.

[14] E. Bertoft, A.K. Avall, J. Inst. Brew., 98 (1992) 433-437.

[15] S. Pérez, A. Imberty, Carbohydr. Eur., 15 (1996) 17-21.

[16] J. Jane, K. Wong, A.E. McPherson, Carbohydr. Res., 300 (1997) 219-227.

[17] G.T. Oostergetel, E.F.J. van Bruggen, Carbohydr. Polym., 21 (1993) 7-12.

[18] O.B. Wurzburg (Ed.), Modified Starches: Properties and Uses, CRC Press, Boca Raton, FL, 1986.

[19] D.J. Gallant, B. Bouchet, P.M. Baldwin, Carbohydr. Polym., 32 (1997) 177-191.

[20] Y.E.M. van der Burgt, J. Bergsma, I.P. Bleeker, P.J.H.C. Mijland, A. van der Kerk-van Hoof, J.P. Kamerling, J.F.G. Vliegenthart, Carbohydr. Res., 312 (1998) 201208.

[21] J.P. Robin, C. Mercier, F. Duprat, R. Charbonnière, A. Guilbot, Cereal Chem., 51 (1974) 389-406.

[22] K. Kainuma, D. French, Biopolymers, 10 (1971) $1673-$ 1680.

[23] T. Komiya, S. Nara, Starch/Stärke, 38 (1986) 9-13.

[24] P.J. Jenkins, A.M. Donald, Starch/Stärke, 49 (1997) $262-267$.

[25] T.A. Scott, E.H. Melvin, Anal. Chem., 25 (1953) 16561661.

[26] J.P. Kamerling, J.F.G. Vliegenthart, in A.M. Lawson (Ed.), Clinical Biochemistry - Principles, Methods, Applications, Vol. 1, de Gruyter, Berlin, 1989, pp. 176-263.

[27] P.W. Arisz, H.J.J. Kauw, J.J. Boon, Carbohydr. Res., 271 (1995) 1-14.

[28] P. Mischnick, G. Kühn, Carbohydr. Res., 290 (1996) 199-207.

[29] I. Ciucanu, F. Kerek, Carbohydr. Res., 131 (1984) 209217.

[30] R.W. Kerr, US patent 2,858,305 (1958). 